\title{
Research and Application of Key Techniques for Preserving and Sterilizing Preprocessed Food Processing
}

\author{
Dong Tianfei \\ Chifeng Industrial Vocational Technical College, Chifeng City, Inner Mongolian Autonomous Region, China, 024000
}

\begin{abstract}
During the development of pre-made and conditioned foods, the corresponding recipes and process requirements should be met, large-scale production work should be performed in the central kitchen, and then distributed to the terminal stores, so that the conditioned foods become more popular. Based on previous work experience, this paper summarizes the research status of key technologies for preserving and sterilizing pre-processed foods. At the same time, this article discusses the key technical application content of pre-conditioned food processing quality and sterilization from four aspects: the application of ultra-high pressure technology, the prevention and control of common microorganisms, the application of tea polyphenols, and the development trend of pre-conditioned food.
\end{abstract}

\section{Introduction}

In recent years, with the development of my country's industrial and agricultural modernization, the simultaneous progress of the "five modernizations" has been rapidly promoted. Coupled with the improvement of the pace of globalization of life, people's demand for catering has also undergone profound changes. Some traditional foods that are ready to eat out of the bag, as well as pre-made and prepared foods, have developed rapidly. As early as 2015, the output value of my country's instant food exceeded 500 billion yuan, and it will grow at a rate of $30 \%$ per year. From this we found that the research on the preserving and sterilizing technology of pre-processed food processing is particularly important, and the relevant staff should increase the degree of attention.

\section{Overview of Pre-prepared and Prepared Food}

In the development of pre-conditioned foods, the main content includes agricultural, livestock, poultry and aquatic products, after proper processing and processing, storage and transportation in the form of frozen or refrigerated, and ultimately sold to consumers. In foreign countries, people use Prepared foods to represent such products, which also involves some ready-to-eat products, such as salads, sauces and so on. In addition, some researchers like to use HMR to name such products, which means that they need to be heated before eating. At this stage, with the continuous improvement of people's living standards, pre-made conditioning products have won the recognition and favor of many consumers. Throughout the entire pre-conditioned food, there are many types of food involved, such as meat, aquatic pre-conditioned and noodles. Due to the different types of pre-conditioned food, the microorganisms involved are also quite different, which requires the staff to pay special attention.

\section{Research Status of Key Technologies for Preserving Sterilization of Processed Foods}

\subsection{Study on the Conditioning of Red Crispy Chicken Dishes}

It can be seen from the previous research that people have specially studied the extension of refrigerated shelf life and the improvement of reheat quality of red crispy chicken dishes. A certain amount of nano cloves and nano calcium oxide are added to it, and then an effective ultrasonic treatment operation is performed vacuum package it after it is out. The subsequent optimal refrigerating temperature of the dish is $4^{\circ} \mathrm{C}$, and the staff will then perform alternate reheating operations of radio frequency and microwave. The characteristic is that due to the application of the above measures, the high temperature and high pressure sterilization step is successfully avoided, otherwise, the quality of the anghong crispy chicken dishes is easily compromised. When refrigerating, if you use radio frequency or microwave for alternating heating, the actual radio frequency penetration will also be greatly improved, strengthening the internal reheating speed. After the above work is completed, the staff must also take low-frequency reheating means to strengthen the reheating flavor on the surface of the dishes. With this reheating means, the actual color and fragrance 
preservation rate can be as high as $94 \%$.

\subsection{Ultraviolet and Nano-zinc Oxide Combined Sterilization}

This kind of sterilization operation can play a role in vegetarian dishes. The specific operation process is as follows: After the raw vegetables are cleaned, the staff should perform the scalding and bleaching enzyme operation, and then fry according to the actual recipe. Then, the nano-zinc oxide, which is a bacteriostatic agent, is added, and the specific packaging form is vacuum packaging, which is stored at room temperature after being sterilized by ultraviolet rays. In this process, nano-zinc can inhibit the rapid growth of microorganisms, and then show the role of microbicides. The antibacterial performance of nano-zinc will be steadily improved by the implementation of ultraviolet sterilization, and it will show a certain synergistic effect, so that the product is under a strong shelf life, and the characteristics of simple operation and energy saving are presented.

\subsection{Stored Dishes at Room Temperature}

This method can play a role in the pickled fish processing method. In the production process of sauerkraut fish, the main raw materials are fresh fish and sauerkraut. After raw material processing and acid adjustment and seasoning, etc., a low-intensity sterilization and cooling process is performed to achieve the form of storing and packaging sauerkraut fish products at room temperature. With the help of this kind of operation, sauerkraut fish products can retain the original flavor and nutrition, and will not add any ski preservatives during processing, and the shelf life can be extended. The researchers said that with the help of traditional low-temperature heat sterilization and ultra-high pressure sterilization, the quality of sauce beef can change. If the staff can apply $400 \mathrm{MPa}, 10 \mathrm{~min}$ ultra-high pressure treatment products, improve their sensory score value, and at the same time help staff achieve food sterilization in a short period of time.

\subsection{Clear Sensory Quality}

The actual sensory quality is mainly related to hardness, $\mathrm{PH}$ value and acid value. The results showed that after the temperature reached $121^{\circ} \mathrm{C}$, the physical and chemical indicators of the roasted whole sheep treated for 20 minutes were better than those of the other treatment groups during the 40 -day storage period. The most obvious increase was the total number of colonies and TVB-N indicators. Similar in quality to freshly roasted whole lamb. Researcher Liu Qinhua made it clear that when the pressure range is in the range of 200 to 600 $\mathrm{MPa}$, the holding time is 10 to $30 \mathrm{~min}$. Under such conditions, the storage performance of roast chicken was studied. Studies have shown that with the continuous increase in pressure intensity and the duration of the pressure holding time, the total amount of microorganisms has dropped significantly, and the growth rate during storage has also slowed down significantly. In addition, the researchers also studied the effect of different pressurization forms on the storage characteristics of roast chicken. The results showed that when the storage conditions were $20^{\circ} \mathrm{C}$, the storage period of roast chicken was 5 to 10 days longer than that without similar treatment under the effect of gradient pressure increase and pulsation pressure increase. In general, the actual pre-prepared and conditioned food work should be adapted to the rhythm of modern society, showing the characteristics of long cooking time and low standardization of traditional Chinese dishes. This can not only meet the needs of modern consumers for food nutrition and deliciousness, but also have a broad market development prospect.

\section{Application Content of Key Technologies for Preserving and Sterilizing Preprocessed Food Processing}

\subsection{Application of Ultra-high Pressure Technology}

\subsubsection{Application of Ultra-high Pressure Technology in Raw Material Processing}

Many foods are mainly made of raw meat during the production process, and are easily affected by microbial infection during processing, which leads to deterioration problems. Ultra-high pressure belongs to the representative of cold sterilization technology, which can eliminate all treatment microorganisms without affecting the quality of raw materials, strengthen the nutritional level of raw materials, and create favorable conditions for subsequent processing and processing operations. However, it can be seen from the actual application process that the staff need to grasp the best parameters such as pressure and processing time to determine the best process plan.

\subsubsection{Application of Ultra High Pressure Technology in Conditioning Processing}

It can be seen from the production and processing of pre-conditioned meat products that the tenderness of meat is an important indicator in the process of sensory quality measurement. If the product can be in a good qualified state, the chewability and taste will also be well guaranteed. More importantly, there is a direct relationship between the tenderness of the meat and the texture of the meat, which is also determined by the protein structure. The characteristics of ultra-high pressure physical modification are mainly to ensure that the water retention of semi-finished materials and the spatial structure and function of proteins will not be affected in any way. In this case, the water state and protein structure will also change, ensuring that the cost, taste and nutritional value will not be affected manually. 


\subsubsection{Application of Ultra High Pressure Technology in Packaging Processing}

In the food processing process, packaging processing is the last operation. At this time, the requirements for sterilization technology are also high. When the actual processing is completed, the food conditioning process is extremely prone to produce pathogenic microorganisms. Many researchers have conducted in-depth research on the ultra-high pressure technology sterilization at this stage to determine the optimal pressure and pressure test time and other parameters, creating favorable conditions for the subsequent industrial application of ultra-high pressure technology. In addition, high pressure will also cause significant changes in cell morphology and structure, rsearcher park used a microscope to carefully observe the spore cell wall and cell membrane under high-pressure environment to clarify the specific separation process. At the same time, there are many researchers who use the model calculation form to present the mechanical stress characteristics of cells under high pressure, which provides favorable conditions for the follow-up work.

\subsection{Common Microorganisms and their Prevention and Control}

\subsubsection{Common Microbes in Pre-conditioned Meat Food}

In general, the development of pre-prepared and prepared meat products in my country is extremely fast and there are many varieties. However, it can be seen from the actual work process that due to some problems in the sterilization of meat products in pre-conditioning in my country, the cold chain system is not perfect, and the shelf life is greatly shortened. This is also the fundamental reason for limiting the development of pre-conditioning in my country. In contrast, there are many types of microorganisms that are prone to pollution problems in pre-conditioned meat prepared foods. These are directly related to the source of meat and the hygienic conditions of processing. In general, the common types of microorganisms in meat and meat products include pseudomonas, acinetobacter, aeromonas, bacillus, leuconostoc, etc. It is precisely because of the obvious complexity and diversity of the microbial flora in the pre-conditioned meat preparations, the types of dominant bacteria are also different in different storage periods. For example, in halal lamb conditioning, enterobacteriaceae represents the dominant spoilage bacteria. At the end of the shelf life, the total number of colonies can reach $69 \%$ of the total bacterial population. For example, Zhejiang Province carried out 235802 batches of samples of 34 kinds of food in the province in 2019. The survey results are shown in Table 1, in which the unqualified rate of frozen food is $23 \%$, the meat product is $36 \%$, and the canned food is $17 \%$.
Table 1 Results of inspection of some food samples in Zhejiang province in 2019

\begin{tabular}{cc}
\hline Food category & Percentage(\%) \\
\hline Meat products & 36 \\
Frozen food & 23 \\
Canned & 17 \\
Seasoning & 22 \\
\hline
\end{tabular}

Common Microorganisms in Aquatic Pre-conditioned Food

There are many types of microorganisms in pre-conditioned aquatic food. Due to the different types of aquatic animals and the living environment, there are some differences in the processing methods and packaging forms involved. For example, in the process of cold storage of fish, the main types of spoilage bacteria are pseudomonas spp., F. thermophila, aeromonas spp., etc. However, from the perspective of practical work, at this stage, the separation and culture forms that people adopt are mainly traditional separation and culture, such as PCR-DGGE and other methods to perform firm work of microorganisms. At this time, there are many uncertainties in the analysis of microbial predominant flora and taxa. In addition, when the aquatic animals were just captured, there was some microbial contamination. During the storage period, the actual storage conditions and the products themselves were easily affected by the physical and chemical properties, which caused a large change in the flora structure. Relevant research shows that the cold-stored salmon in the early storage period is mainly affected by the genus vitis and pseudomonas, which are also known as specific spoilage bacteria.

Common Microbes in Pre-made and Conditioned Food of Noodles

The main contents involved in the pre-made and conditioned food of noodles and rice are quick-frozen dumplings, rice balls, spores, steamed buns, rice, etc. In the environment with high temperature, microbial contamination of quick-frozen food is very serious. For example, in quick-frozen dumplings, microorganisms are mainly present in the filling, and rice dumpling microorganisms are mainly concentrated in the fabric. The inspection of a brand of quick-frozen dumplings showed that the bacteria present in the quick-frozen dumplings were streptococcus, pseudomonas and so on. It should be noted that quick-frozen dumplings are repeatedly frozen and thawed many times, which affects the physical and chemical quality of the product, but there is not much correlation between microbial indicators such as the total number of colonies and coliform bacteria. Therefore, people need to pay more attention to the research of common microbes in rice-based pre-made and conditioned food.

Application of Tea Polyphenols

Tea polyphenols are a kind of natural food additives. The content in green tea is relatively high, and the actual proportion can reach $36 \%$. This is also the focus of research on green tea polyphenols at home and abroad. Tea polyphenols can show good activity in pre-conditioned foods, and the most important one is its 
antioxidant function. In the process of food preservation, tea polyphenols can also inhibit the oxidation of fat and protein, providing a better preservation environment for food. For example, in pre-conditioned meat prepared foods, staff can add tea polyphenols with mass concentrations of $0,0.02,0.2$, and $1.0 \mathrm{mg} / \mathrm{mL}$ under oxidizing conditions to clarify the physical and chemical effects of meat fibrillin. The results show that through this treatment operation, the mass concentration of tea polyphenols will increase, the water retention of the gel will also show an upward trend, and the free water content will also show a downward trend.

\subsection{The Development Trend of Prepared Food}

Through the implementation of pre-made and conditioned food work, the flavor and nutritional value of the dishes can be effectively protected, and it will also have a great impact on the production and reproduction of pathogenic microorganisms. However, due to the low acidity in Chinese dishes, if only the ultra-high pressure technology is applied, the economic characteristics cannot be presented. In the later period, it will also cooperate with the long-term cold chain storage and transportation operations. It is precisely because of the above problems that people have focused their research on the cooperation of ultra-high pressure technology and other sterilization technologies. In general, the quality and sterilization technology of pre-processed and regulated food processing in my country needs to be further improved, and the maintenance and processing technology is compatible with the development trend of the new era.

\section{Conclusion}

In summary, it can be seen from the application of key technologies for preserving and sterilizing pre-processed food processing, relevant staff need to understand the cooking time and standardization characteristics of traditional Chinese dishes, to ensure the commercialization of dishes and the global marketing goals of marketing, and then to the market development space and growth potential are presented. With the continuous development of my country's food industry, many new technologies can be applied in food production to avoid more food safety problems.

\section{References}

1. Li Na, Cai Lufeng. Conditioning food and its development status and trend [J]. Food and Food Industry, 2020,27(01):31-34.

2. Mao Pengquan, Yu Qundi, Zheng Linbo. Dynamics of Chinese tube whip shrimp prepared foods to predict product shelf life [J]. Journal of Zhejiang Ocean University (Natural Science Edition), 2019, 38(05): 422-428.

3. Zhao Juyang, Wang Meng, Shi Changbo. Development and quality research progress of pre-prepared and prepared foods in dishes[J]. Chinese Condiments, 2019, 44(08): 193-196.

4. Zhang Qun. Research and application of key technologies for preserving, preserving and sterilizing food processing $[\mathrm{J}]$. Journal of Food and Biotechnology, 2019, 38(03): 160.

5. Zhang Qun. Microwave field conditioning and drying technology for synergistic regulation of food[J]. Journal of Food and Biotechnology, 2018, 37(10): 1120. 